\title{
22 Reizdarmsyndrom
}

Robert Ehehalt

\subsection{Kapitelzusammenfassung}

Das Reizdarmsyndrom (RDS) ist die häufigsten funktionelle Gesundheitsstörung in der Magen-Darm-Praxis. Es ist eine Ausschlussdiagnose. Relevante Differenzialdiagnosen, wie z. B. eine chronisch entzündliche Darmerkrankung, sollten vor der Diagnose möglichst sicher ausgeschlossen sein. Die Symptomatik des RDS ist individuell stark variierend. Zu den Hauptsymptomen gehören abdomineller Schmerz, Obstipation, Diarrhö, Stuhl-Drangsymptomatik und Blähungen. Nach den Hauptsymptomen werden verschiedenen Untergruppen definiert. So kann nach den aktuellen Empfehlungen das RDS in eine Obstipation-prädominante (RDS-O), eine Diarrhoeprädominante (RDS-D) sowie eine gemischte (RDS-M) Form unterschieden werden. Im klinischen Alltag wird oft zusätzlich noch der Reizdarm vom „Bläh-Typ“ von diesen Gruppen abgegrenzt. Aufgrund der klinischen Variabilität gibt es keine definierte Standardtherapie, sondern die Behandlung erfolgt individuell, vom Schweregrad abhängig und symptomorientiert. Therapeutische Ansätze sind neben der ärztlichen Führung (psychosomatische Grundversorgung), Hilfen zur Veränderung des Ernährungs- und Lebensstils, diverse Phytotherapeutika, Probiotika, Ballaststoffe, Laxantien, Spasmolytika, Prokinetika sowie bei schwereren Formen auch Antidepressiva oder Neuroleptika.

\subsection{Definition und Klassifikation}

Das Reizdarmsyndrom (RDS) zählt zu den funktionellen Magen-Darm-Erkrankungen. In den allgemeinen Diagnoseverfahren sind keine strukturellen Ursachen nachzuweisen. Seit längerem wird daher versucht, das Reizdarmsyndrom anhand von charakteristischen klinischen Beschwerden zu definieren. Dies ist aber wegen der Vielfältigkeit, Individualität und Wechselhaftigkeit der Beschwerden schwierig. So können die bisherigen Definitionen von Manning et al., Kruis et al. und die seit 1990 mehrfach überarbeiteten Rom-Kriterien (zuletzt 2016) die klinische Realität nicht ausreichend abdecken und es bleibt fraglich, inwieweit eine Eingruppierung des Reizdarmsyndroms anhand von Symptomen überhaupt möglich ist. Diese Klassifikationen sind daher eher wissenschaftlichen Studien vorbehalten und spielen in der allgemeinen Praxis kaum eine Rolle. Die in der Rom-Klassifikation empfohlen Subtypen des RDS sind in Tab.22.1 aufgeführt. Im klinischen Alltag wird aus therapeutischen Überlegungen heraus zusätzlich oft noch der Reizdarm vom Bläh-Typ davon abgegrenzt. 
Tab. 22.1: Untergruppen des Reizdarmsyndroms nach Rom.

\begin{tabular}{ll}
\hline RDS-0 (Obstipations-Typ) & $\begin{array}{l}\text { Harter Stuhlgang bei } \leq 25 \% \text { aller Defäkationen und flüssiger } \\
\text { Stuhlgang }<25 \% \text { aller Defäkationen }\end{array}$ \\
\hline RDS-D (Diarrhoe-Typ) & $\begin{array}{l}\text { Flüssiger Stuhlgang bei } \leq 25 \% \text { aller Defäkationen und harter } \\
\text { Stuhlgang }<25 \% \text { aller Defäkationen }\end{array}$ \\
\hline RDS-M (Misch-Typ) & $\begin{array}{l}\text { Harter Stuhlgang bei } \leq 25 \% \text { aller Defäkationen und flüssiger } \\
\text { Stuhlgang } \leq 25 \% \text { aller Defäkationen }\end{array}$ \\
\hline
\end{tabular}

In der immer noch neusten Leitlinie der DGVS (Deutsche Gesellschaft für Verdauungs- und Stoffwechselkrankheiten) von 2011 wird aufgrund der o. g. Überlegungen auf eine definierende Symptomkombination verzichtet und aus pragmatischen Gründen das RDS lediglich anhand von drei Kriterien definiert:

1. Bauchbeschwerden: Dies beinhaltet sämtliche anhaltenden Darmbeschwerden (>3 Monate), wie z. B. Bauchschmerzen und Blähungen, die von Patienten und Arzt auf den Darm bezogen werden.

2. Patient leidet signifikant: Dies bedeutet, dass die Beschwerden dazu führen, dass die Lebensqualität relevant beeinträchtigt wird und der Patient medizinische Hilfe sucht.

3. Ausschluss anderer Erkrankungen: Andere Erkrankungen, die sich mit ähnlichen Beschwerden manifestieren können, müssen möglichst verlässlich ausgeschlossen sein.

\section{3 Ätiologie und Pathogenese}

Die Pathogenese des Reizdarmsyndroms ist vielschichtig und weiterhin weitgehend unverstanden. Einig ist man sich allerdings über eine Art Bio-Psycho-Soziales Modell, was bedeutet, dass ein Wechselspiel von psychosozialen und biologischen Faktoren bei der Pathogenese eine signifikante Rolle spielt.

Für das Postinfektiöse Reizdarmsyndrom (PI-RDS), welches nach einer akuten infektiösen Darmerkrankung auftreten kann, gibt es allerdings epidemiologische Daten, die einen kausaler Zusammenhang zeigen. Nach einer gastrointestinalen Infektion entwickeln 7-36 \% der Erkrankten postinfektiös ein RDS, nach einer Reisediarrhö tritt ein RDS bei 4-14\% der betroffenen Personen auf. Risikofaktoren für das PI-RDS sind weibliches Geschlecht, Rauchen, Dauer und Schwere der infektiösen Entzündung, jüngeres Alter (<60 Jahre) und das gleichzeitige Vorliegen einer psychischen Erkrankung (z. B. Depression oder Hypochondrie).

Generell geht man von einem multifaktoriellen Geschehen aus, welches von Patient zu Patient variiert. Bei jedem Betroffenen trägt wahrscheinlich eine andere 
Konstellation aus Umweltfaktoren sowie molekulare und zelluläre Mechanismen zur Pathogenese bei, welche sich gegenseitig verstärken können.

Als ursächlich werden beispielsweise eine Störung der viszeralen Sensitivität, eine verringerte intestinale Barrierefunktion, eine Störung der gastrointestinalen Motilität oder eine Veränderung des Microbioms (Dysbiose) diskutiert. Außerdem konnten genetische Prädispositionen und in Einzelfällen auch eine Veränderung der Immunaktivierung nachgewiesen werden. Im nicht unerheblichen Umfang spielen auch psychische bzw. psychiatrische Störungen, psychosoziale Stressoren und diverse Umweltfaktoren eine wichtige Rolle.

\subsection{Prävalenz und Inzidenz}

Die Prävalenz des RDS ist trotz unterschiedlicher kultureller Einflüsse weltweit in etwa vergleichbar. Je nach zugrundeliegenden Diagnose-Kriterien (Manning, Kruis, Rom I-III) liegt sie bei 2,5 bis $25 \%$. Es besteht außerdem wahrscheinlich noch eine hohe Dunkelziffer, da vermutlich wegen des benignen und wechselnden Charakters der Erkrankung nur ein Teil der Betroffenen mit RDS-Beschwerden sich in ärztlicher Behandlung befindet. Es sind im Allgemeinen mehr Frauen als Männer betroffen.

\subsection{Diagnose und Differentialdiagnose}

Die Diagnose des Reizdarmsyndroms ist vielschichtig und basiert auf einem Ausschlussverfahren. Um wirtschaftlich vorgehen zu können, wurde in der Deutschen S3-Leitlinie zur Abklärung des Beschwerdebildes ein einfacher Algorithmus empfohlen (Abb. 22.1):

Zur Diagnosestellung sollten danach grundsätzlich drei Kriterien erfüllt sein:

1. Symptomanamnese: Das Muster und das Ausmaß der Beschwerden müssen mit den Diagnosekriterien eines Reizdarmsyndroms vereinbar sein. Dazu gehören:

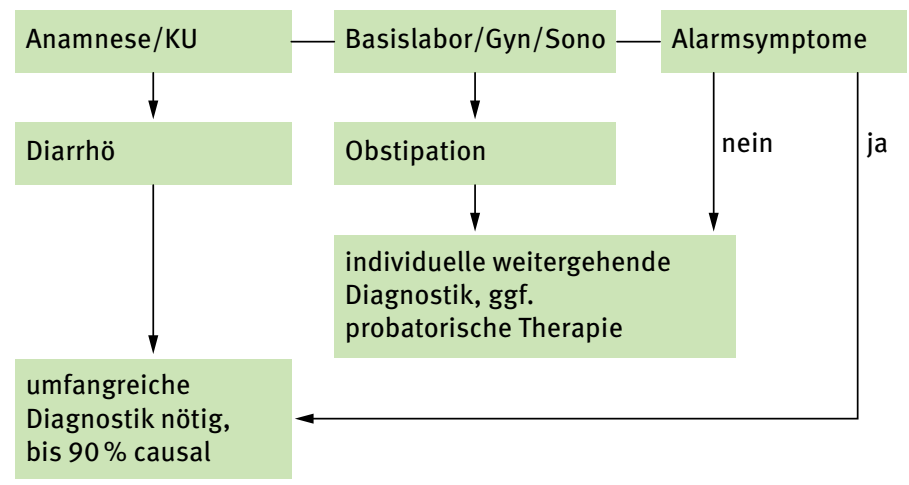

Abb. 22.1: Rationaler Abklärungsalgorithmus (nach DGVS Leitlinie). 
a. abdominale Schmerzen (meist Besserung durch Stuhlgang),

b. eine veränderte Stuhlform mit mindestens zwei der drei folgenden Aspekte:

- Frequenz (wie häufig, zu selten, zu oft),

- Konsistenz (hart, breiig, wechselnd),

- Passage (mühsam, gesteigerter Stuhldrang, inkomplette Darmentleerung, Schleimbeimengung),

c. das Gefühl der abdominalen Distension oder

d. allgemein Blähungen.

2. Ausschluss von Alarmsymptome: Das Fehlen von Alarmsymptomen („red flags“) ist für die Diagnosestellung des RDS zunächst Voraussetzung. Alarmsymptome weisen auf eine organische Ursache hin, die intensiv abgeklärt gehört. Zu den Alarmsymptomen zählen u.a. Fieber, Blut im Stuhl, ein erhöhter inflammatorischer Stuhlmarker, eine Anämie, stetige Zunahme der Beschwerden, Gewichtsverlust, nächtliches Erwachen aufgrund der Beschwerden sowie schwere anhaltende Diarrhoen. Außerdem gilt eine signifikante Veränderung der bisherigen Darmbeschwerden als ein alarmierendes Zeichen. Alarmsymptome, welche eher auf eine organische Ursache hinweisen, sind in Tab. 22.2 gezeigt:

Tab. 22.2:

- Gewichtsverlust

- Fieber

- Anämie, Blut im Stuhl

- Stetige Zunahme der Beschwerden

- Nächtliches Erwachen wegen der Beschwerden

- Anhaltende (schwere) Diarrhoe

- Symptombeginn im Alter > 50 Jahre

- Signifikante Veränderung der Bauchbeschwerden

- Erhöhte inflammatorische Stuhlmarker (Calprotectin, Lactoferrin)

3. Differentialdiagnostisches Sichern der Diagnose RDS: Bevor die Diagnose eines RDS gestellt werden kann, müssen relevante Differentialdiagnosen ausgeschlossen sein. Einige relevante Differentialdiagnosen sind in Abhängigkeit ihres Leitsymptoms in Tab. 22.3 aufgeführt.

Bei jedem Patienten muss unbedingt eine Basisdiagnostik durchgeführt, mit der bereits viele Differentialdiagnosen ausgeschlossen werden können. Dies ist wichtig, da einige der diese Diagnosen lebensbedrohlich sein können. 
Tab. 22.3: Auswahl an Differentialdiagnosen in Abhängigkeit des Leitsymptoms.

\begin{tabular}{|c|c|}
\hline $\begin{array}{l}\text { Reizdarmsyndrom- } \\
\text { Leitsymptom }\end{array}$ & Differentialdiagnosen (Auswahl) \\
\hline Diarrhoe & $\begin{array}{l}\text { - infektiöse Kolitis durch Viren, Bakterien und Parasiten (z. B. C. diffici- } \\
\text { le, CMV, Histoplasmose) } \\
\text { - chronisch entzündliche Darmerkrankungen (M. Crohn, Colitis ulcerosa) } \\
\text { - Zöliakie } \\
\text { - bakterielle Fehlbesiedlung (SIBO) } \\
\text { - tropische Sprue/M. Wippel } \\
\text { - Nahrungsmittelunverträglichkeiten (z. B. Laktose- und Fruktosein- } \\
\text { - toleranz) } \\
\text { - Nahrungsmittelallergien } \\
\text { - mikroskopische Kolitis } \\
\text { - Pankreasinsuffizienz } \\
\text { - autonome Neuropathie (Diabetes) } \\
\text { - Medikamentenunverträglichkeit } \\
\text { - Inkontinenz } \\
\text { - Hyperthyreose } \\
\text { - paradoxe Diarrhö (z. B. Kolorektales Karzinom) } \\
\text { - hormonaktives NET }\end{array}$ \\
\hline Schmerz & $\begin{array}{l}\text { - M. Crohn } \\
\text { - gastroduodenale Ulzeration } \\
\text { - Mesenterialischämie } \\
\text { - Tumoren } \\
\text { - Porphyrie } \\
\text { - Endometriose } \\
\text { - Verwachsungen } \\
\text { - Ovarialtumoren } \\
\text { - viszerale Tuberkulose } \\
\text { - Mittelmeerfieber } \\
\text { - chronische Appendizitis }\end{array}$ \\
\hline Obstipation & $\begin{array}{l}\text { - strukturelle Stuhlentleerungsstörung } \\
\text { - Medikamentennebenwirkung } \\
\text { - Divertikulose } \\
\text { - stenosierender Prozess (z. B. Kolorektales Karzinom) } \\
\text { - Hypothyreose } \\
\text { - strukturelle Motilitätsstörungen (z. B. CIPO) }\end{array}$ \\
\hline Blähungen & $\begin{array}{l}\text { - bakterielle Fehlbesiedlung (SIBO) } \\
\text { - Nahrungsmittelunverträglichkeiten (z. B. Laktose- und Fruktosein- } \\
\text { toleranz) } \\
\text { - Pankreasinsuffizienz } \\
\text { - Verwachsungen } \\
\text { - strukturelle Motilitätsstörungen }\end{array}$ \\
\hline
\end{tabular}


Zur Basisdiagnostik gehören neben der ausführlichen Anamnese, die körperliche Untersuchung sowie ein Basis-Labor, wozu primär ein Blutbild, CRP oder BSG, Pankreas-, Leber- und Nierenwerte, Blutzucker sowie ein Urinsediment gehören (Tab. 22.4). Eine psychosoziale Anamnese ist wichtig. Eine abdominale Sonographie sollte als Screening durchgeführt werden.

Calprotectin, Lactoferrin oder ein anderer inflammatorischer Stuhlmarker sollte bestimmt werden, um das RDS von einer entzündlichen Darmerkrannkung zu differnzieren.

In Abhängigkeit von Beschwerdebild und Anamnese werden weitere Untersuchungen wie z. B. TSH, Transglutaminase-AK, IgA sowie Calcium und Phosphat (Ausschluss Hyperparathyreoidismus) (Tab. 22.4) durchgeführt.

Eine Bestimmung des Eisenstatus (Ferritin oder Transferrinsättigung) kann sinnvoll sein. Ein latenter Eisenmagel weist zunächst eher auf eine organische Ursache hin.

Tab. 22.4: Laboruntersuchungen zum Ausschluss von RDS Differentialdiagnosen.

Generell empfohlen (Basislabor)

Blutbild

BSG oder CRP

Leber- und Pankreasenzyme

Serum-Elektrolyte, Retentionswerte

Glukose (nüchtern)

Urinstatus

Calprotectin im Stuhl

Gegebenenfalls weiterführendes Labor (Auswahl)

Ferritin oder Transferrinsättigung

TSH

Transglutaminase-Antikörper

IgA

Stuhl-Mikrobiologie

Calcium, Phosphat

Vitamin D

Parathormon intakt (iPTH)

Bei Frauen sollte eine gynäkologische Untersuchung veranlasst werden, da z. B. Ovarialtumoren im Frühstadium typische Reizdarmsymptome erzeugen können.

Eine weiterführende Diagnostik hängt von der gezielteren Fragestellungen ab. Es ist sinnvoll, zwischen Patienten mit und ohne Durchfallbeschwerden zu unterscheiden.

Liegt als Hauptsymptom ein Durchfall vor, ist eine eingehende diagnostische Abklärung einschließlich Erregerdiagnostik im Stuhl, endoskopischer (Gastroskopie mit tiefen Duodenalbiopsien sowie Koloskopie mit Stufenbiopsien) und funktions- 
diagnostischer Untersuchungen (wie z.B. H2-Atemtests auf Laktose, Fruktose und Sorbit) empfohlen.

Besteht keine wesentliche Diarrhö ist eher eine individuell abgestimmte Diagnostik notwendig. Auch hier können eine Ileokoloskopie, eine Ösophago-GastroDuodenoskopie, spezielle Funktionstests (z. B. $\mathrm{H}_{2}$-Atemtest), manometrische und bildgebende Verfahren sinnvoll sein.

\subsection{Therapie}

Beim RDS ist es insbesondere wegen der intraindividuellen Variation des klinischen Krankheitsbildes schwierig, einen wirklichen Standard zu definieren. Es gilt daher allgemein eine individualisierte, Schweregrad-gestufte Therapie bezogen auf das jeweilige Leitsymptom und dem individuellen biopsychosozialen Modell durchzuführen (Tab. 22.5).

Tab. 22.5: Therapie des Reizdarmsyndroms unter Beachtung von Schweregrad und Leitsymptomatik (Therapieauswahl).

\begin{tabular}{|c|c|c|c|c|c|}
\hline \multicolumn{2}{|c|}{ Verlauf/Schweregrad } & Diarrhö & Obstipation & Blähungen & Schmerzen \\
\hline leicht & $\begin{array}{l}\text { ärztliche } \\
\text { Führung }\end{array}$ & \multicolumn{4}{|c|}{$\begin{array}{l}\text { Aufklärung (beruhigen, Diagnose festlegen und mitteilen), Psycho- } \\
\text { edukation (Entwicklung eines individuellen biopsychosozialen Er- } \\
\text { klärungsmodells), vertrauliche Arzt-Patienten-Beziehung }\end{array}$} \\
\hline & $\begin{array}{l}\text { allgemeine } \\
\text { Maßnahmen }\end{array}$ & \multicolumn{4}{|c|}{ Entspannungsverfahren, Stressmanagement, Ressourcen Aktivierung } \\
\hline & $\begin{array}{l}\text { Ernährung/ } \\
\text { Lebensstil }\end{array}$ & \multicolumn{4}{|c|}{$\begin{array}{l}\text { Ernährungstherapeutische Beratung, Tagesstruktur, Genussmittel, } \\
\text { Bewegung/Sport }\end{array}$} \\
\hline & Probiotika & \multicolumn{4}{|c|}{$\begin{array}{l}\text { Z. B. E. coli Nissle, B. infantis, B. animalis, L. casei Shirota, B. bifidum, } \\
\text { L. plantarum }\end{array}$} \\
\hline & Phytotherapie & \multicolumn{4}{|c|}{$\begin{array}{l}\text { z. B. STW5, Flohsamenschalen, Chiasamen, Pfefferminzöl, Papaya- } \\
\text { Extrakt }\end{array}$} \\
\hline \multirow[t]{2}{*}{ mittel } & $\begin{array}{l}\text { symptom- } \\
\text { orientierte } \\
\text { Medikation }\end{array}$ & $\begin{array}{l}\text { Cholestyramin } \\
\text { Loperamid } \\
\text { Racecadotril } \\
\text { Quellmittel }\end{array}$ & $\begin{array}{l}\text { Macrogol } \\
\text { Laxanzien } \\
\text { Prucaloprid } \\
\text { Linaclotid }\end{array}$ & $\begin{array}{l}\text { Entschäume } \\
\text { Rifaximin } \\
\text { Pfefferminz } \\
\text { Kümmelöl } \\
\text { Rotierende } \\
\text { Antibiose }\end{array}$ & $\begin{array}{l}\text { Butylscopolamin } \\
\text { Mebeverin } \\
\text { Metamizol } \\
\text { andere } \\
\text { Analgetika }\end{array}$ \\
\hline & Psychotherapie & \multicolumn{4}{|c|}{$\begin{array}{l}\text { z. B. Bauchhypnose, CTB (kognitive Verhaltenstherapie), Psycho- } \\
\text { therapie }\end{array}$} \\
\hline schwer & $\begin{array}{l}\text { Antidepressiva/ } \\
\text { Neuroleptika }\end{array}$ & \multicolumn{4}{|c|}{ z. B. Amitriptylin, Cymbalta, SSRI } \\
\hline
\end{tabular}


Ein nicht unerheblicher Aspekt ist den Patienten darüber aufzuklären, was ein RDS bedeutet. Der Arzt sollte dem Patienten ein biopsychosoziales Erklärungsmodell für seine Erkrankung erläutern. Psychische Schulungsmaßnahmen und Informationen über den Verdauungstrakt, den benignen Charakter der Erkrankung, therapeutische Optionen, psychophysiologische Zusammenhänge (,gut-brain axis“) oder die Bedeutung des Mikrobioms sind sinnvoll und die Erläuterungen dazu haben bereits einen therapeutischen Charakter. Bei milden Beschwerden kann die Aufklärung über das Krankheitsbild und die Beruhigung des Patienten therapeutisch ausreichend sein.

Die medikamentöse Therapie ist wegen der Variabilität des klinischen Erscheinungsbildes immer individuell festzulegen. Nach Möglichkeit sollte sie sich an dem biopsychosozialen Erklärungsmodell orientieren.

Die Pathogenese ist nicht ausreichend verstanden und es gibt daher keine kausale Behandlung oder Standardtherapie. Jeder medikamentöse Therapieversuch hat daher immer auch einen empirischen Charakter.

Die medikamentösen Ansätze orientieren sich im Wesentlichen an den Hauptsymptome Durchfall, Verstopfung, Blähung und Schmerzen (Tab. 22.5).

Bei Verstopfungsbeschwerden wird wie bei der chronischen Obstipation mit einer Stufentherapie vorgegangen. Stufe 1 bedeutet Änderung des Life-Style, Einnahme von Ballaststoffen (z. B. Backpflaumen) oder Magnesium, Sport, ausreichende Flüssigkeitszufuhr und Toilettentraining. Wenn das nicht ausreicht, sollten Quellmittel wie Haferkleie, Flohsamenschalen oder Chiasamen und als nächster Schritt ein komplexes Polymer oder Zuckermolekül (z. B. Macrogol oder Lactulose) verwendet werden. Ist beides nicht erfolgreich, werden Laxantien (z. B. Laxoberal ${ }^{\circledR}$ oder Dulco$\operatorname{lax}^{\circledR}$ ) verwendet. Bei schwerer Obstipation werden die Verfahren kombiniert. Ist auch dieser Schritt nicht ausreichend, kann ein Prokinetikum versucht werden. Hier ist seit einiger Zeit das Prucaloprid (Resolor ${ }^{\circledR}$ ) verfügbar. Es handelt sich um einen Serotonin-(5HT4)-Rezeptor-Agonisten. In Deutschland ist Prucaloprid allerdings nur für die chronische Obstipation und nicht für das RDS zugelassen.

Linaclutid (Constella ${ }^{\circledR}$ ) ist eine weitere Substanz, welche beim RDS vom Obstipationstyp eingesetzt werden kann. Es aktiviert die Guanylatcyclase, was zu einer vermehrten Sekretion von Chlorid und Wasser führt. Außerdem wird Linaclutid eine Herabsetzung der viszeralen Schmerzsensitivität zugeschrieben. In den USA ist es daher für das Reizdarmsyndrom vom Obstipationstyp zugelassen. In Deutschland ist es verschreibungspflichtig, wird aber nicht von den gesetzlichen Krankenkassen übernommen.

Bei abdominellen Schmerzen zeigen Ballaststoffe kaum einen Effekt. Neben den Schmerzmitten wie Novaminsulfon können Spasmolytika (Mebeverin, Butylscopolamin) zur Linderung chronisch rezidivierender Schmerzen eingesetzt werden.

Trizyklische Antidepressiva und SSRI (Selektive Serotonin Reuptake Inhibitoren) können zur Schmerztherapie im Sinne einer veränderten Schmerzverarbeitung verwendet werden. 
Eine unterstützende Wirkung wurde in kleineren Studien den Probiotikastämmen Bifidobacterium infantis, Bifidobacterium bifidum, Lactobacillus casei shirota und Lactobacillus plantarum, E. coli Nissle zugeordnet. So können zum Symptommanagement z. B. Yacult ${ }^{\circledR}$ (Lactobacillus casei Shirota) Kijimea ${ }^{\circledR}$ (Bifidobacterium bifidum) oder Innovall RDS ${ }^{\circledR}$ (Lactobacillus plantarum 299v) versucht werden.

Bei dem Leitsymptom Diarrhö können Quellmittel (Haferkleie, Flohsamenschalen oder Chiasamen) durch Wasserbindung eine erhöhte Passagezeit und eine verbesserte Stuhlkonsistenz ermöglichen.

Ansonsten werden bei diesem RDS-Typ die klassischen Antidiarrhoika (Racecadotril, Loperamid, Cholestyramin) eingesetzt.

Trizyklische Antidepressiva können nach Nutzen-Risiko-Abwägung wegen ihrer motilitätshemmenden Wirkung ebenfalls niedrig dosiert bei RDS-Typ mit Diarrhoe angewendet werden. Auch hier können Probiotika unterstützend helfen.

Die Quellmittel können zu Blähbeschwerden führen, weshalb sie zur Behandlung von Flatulenz oder Meteorismus möglicherweise nicht geeignet sind, es sei denn eine Obstipationskomponente ist mitverantwortlich für die Blähbeschwerden. Für Probiotika wie Lactobacillus plantarum zeigten sich positive Studienergebnisse. Ansonsten kommen beim RDS vom Bläh-Typ symptomatisch Simethicon und Kümmel- und Pfefferminzöle oder deren Extrakte (z. B. IBgard ${ }^{\circledR}$, Carmenthin ${ }^{\circledR}$ ) zur Anwendung. Der Einsatz von Antibiotika zur Symbioselenkung wird auch immer wieder versucht. Insbesondere Rifaximin $\left(\right.$ Xifaxan $^{\circledR}$ ) erscheint nebenwirkungsarm und kann die Symptome verbessern. Rifaximin ist allerdings für das RDS nicht zugelassen.

Bei schweren RDS-Fällen können übergreifend Neuroleptika oder Antidepressiva auch in psychiatrischer Dosierung eingesetzt werden.

Der RDS-Markt ist proliferierend. Neuere, zu beachtenden Möglichkeiten sind die Bauchhypnose, eingesetzt beim Reizdarm mit Schmerzen, das oben erwähnte lokal wirksame Antibiotikum Rifaximin $\left(\right.$ Xifaxan $\left.^{\circledR}\right)$ sowie neue Probiotika, wie z. B. Kijimea $^{\circledR}$ (Bifidobacterium bifidum), Innovall RDS (Lactobacillus plantarum 299v) oder VSL\# $3^{\circledR}$. Auch der FODMAP-Diät oder dem Mikrobiomtransfer (FMT, Stuhltransplantation) werden eine therapeutische Wirkung zugesprochen. 


\section{Weiterführende Literatur}

Craig 0. New therapies in Irritable Bowel Syndrome: what works and when. Curr Opin Gastroenterol. 2018;34(1):50-56.

Drossman DA, Tack J, Ford AC, et al. Neuromodulators for Functional Gastrointestinal Disorders (Disorders of Gut-Brain Interaction): A Rome Foundation Working Team Report. Gastroenterology. 2018;154(4):1140-1171.

Layer P, et al. [Irritable bowel syndrome: German consensus guidelines on definition, pathophysiology and management]. Z Gastroenterol. 2011;49(2):237-293.

Quigley EM, Fried M, Gwee KA, et al. Review Team:.World Gastroenterology Organisation Global Guidelines Irritable Bowel Syndrome: A Global Perspective Update September 2015. J Clin Gastroenterol. 201650(9):704-713.

Simrén M, Tack J. New treatments and therapeutic targets for IBS and other functional bowel disorders. Nat Rev Gastroenterol Hepatol. 2018 Jun 21. doi: 10.1038/s41575-018-0034-5. [Epub ahead of print] 